\title{
A CATEGORIFICATION OF INTEGRAL SPECHT MODULES
}

\author{
MIKHAIL KHOVANOV, VOLODYMYR MAZORCHUK, AND CATHARINA STROPPEL
}

(Communicated by Dan Barbasch)

\begin{abstract}
We suggest a simple definition for categorification of modules over rings and illustrate it by categorifying integral Specht modules over the symmetric group and its Hecke algebra via the action of translation functors on some subcategories of category $\mathcal{O}$ for the Lie algebra $\mathfrak{s l}_{n}(\mathbb{C})$.
\end{abstract}

\section{INTRODUCTION}

In this paper we describe categorifications of irreducible modules for the symmetric group $S_{n}$ and the corresponding (generic) Hecke algebra $\mathcal{H}_{n}$. The category of complex $S_{n}$-modules is semi-simple and the irreducible modules are the so-called Specht modules $\mathcal{S}(\lambda)$, indexed by partitions $\lambda$ of $n$. Analogous statements hold for the Hecke algebra $\mathcal{H}_{n}$, where we denote the Specht module by $\tilde{\mathcal{S}}(\lambda)$. The symmetric group $S_{n}$ is isomorphic to the Weyl group of the semi-simple complex Lie algebra $\mathfrak{s l}_{n}$. Associated to any composition $\mu$ of $n$, there is the corresponding Young subgroup $S_{\mu}$ of $S_{n}$ and the corresponding parabolic subalgebra $\mathfrak{p}_{\mu}$ of $\mathfrak{s l}_{n}$. There is also the partition $\lambda$ associated with $\mu$ (i.e. $\lambda$ coincides with $\mu$ up to reordering of the parts), and the dual partition $\lambda^{\prime}$ of $\lambda$ with the corresponding Specht module $\mathcal{S}\left(\lambda^{\prime}\right)$.

On the other hand, there is the BGG category $\mathcal{O}$ for $\mathfrak{s l}_{n}$ and its direct summand $\mathcal{O}_{0}$ determined by the trivial central character. Let $\mathcal{O}_{0}^{\mu}$ be the full subcategory of $\mathcal{O}_{0}$ consisting of all $\mathfrak{p}_{\mu}$-locally finite modules.

We establish a canonical bijection between the isomorphism classes of indecomposable projective-injective modules in $\mathcal{O}_{0}^{\mu}$ and the Kazhdan-Lusztig basis of $\mathcal{S}\left(\lambda^{\prime}\right)$, defined in $[\mathrm{KL}]$. The category $\mathcal{O}_{0}^{\mu}$ is equivalent to the category of finitely generated $A$-modules, where $A$ is a certain finite-dimensional positively graded Koszul algebra ([BGS]). By working with the category $\mathcal{A}$ of graded finitely generated $A$-modules we obtain a canonical bijection between the isomorphism classes of indecomposable projective-injective modules in $\mathcal{A}$, up to grading shifts, and the Kazhdan-Lusztig basis of $\tilde{\mathcal{S}}\left(\lambda^{\prime}\right)$.

Every Specht module $\tilde{\mathcal{S}}(\lambda)$ has a symmetric, non-degenerate, $\mathcal{H}_{n}$-invariant bilinear form $\langle$,$\rangle with values in \mathbb{Z}\left[v, v^{-1}\right]$, which is unique up to a scalar $([\mathrm{Mu}])$. Under the bijection above, this form can be categorified and becomes the bifunctor

Received by the editors September 14, 2006.

2000 Mathematics Subject Classification. Primary 17B10, 05E10, $20 \mathrm{C} 08$.

The first author was partially supported by the NSF grant DMS-0407784.

The second author was supported by STINT, the Royal Swedish Academy of Sciences, the Swedish Research Council and the MPI in Bonn.

The third author was supported by EPSRC grant 32199 . 
$\operatorname{Hom}_{A}\left({ }_{-}, \mathrm{d}\left({ }_{-}\right)\right)$which takes two graded $A$-modules $M, N$, with $M$ projective, to the graded vector space $\operatorname{Hom}_{A}(M, \mathrm{~d}(N))$, where $d$ is the standard duality functor on $\mathcal{A}$. The bilinear form, evaluated at images of $M$ and $N$ in the Grothendieck group, equals the Hilbert polynomial of this graded vector space.

To make sense of the $\mathcal{H}_{n}$-invariance, we consider an action of the Hecke algebra on the category $\mathcal{A}$ via the so-called translation functors in $\mathcal{O}$, introduced by Jantzen ([Ja], $[\mathrm{BG}])$.

As a result we get a categorification of the Specht modules for both the symmetric group (via the category $A$-mod) and the Hecke algebra (via the category $\mathcal{A})$, where the action of the group ring and the Hecke algebra, respectively, is given by translation functors and the bilinear form is obtained from the bifunctor Hom as briefly explained above. In particular, dimensions of endomorphism rings of projective-injective modules in $\mathcal{O}_{0}^{\mu}$ can be obtained purely combinatorially using the non-degenerate form.

These results can be used to give a categorical version for the original Springer construction of irreducible representations of the symmetric group in terms of the top degree part of the cohomology ring of the Springer fibers; see [St2, Theorem 4.3.5] for details. In particular, we have a natural bijection between the indecomposable projective-injective modules in $\mathcal{O}_{0}^{\mu}$ and the irreducible components of the associated Springer fiber. Our categorical version of the bilinear form $\langle$,$\rangle should$ have an interpretation in terms of the intersection homology Poincaré polynomials of pairwise intersections of irreducible components; see [Fu] and [St2, Theorem 4.6.1] for the special cases of two-row and hook-shape partitions.

We start by giving a definition of a categorification and then we prove the two categorification results with the bilinear form obtained at the end. By a "module" we always mean a right module.

\section{Categorification of modules with integral structure Constants}

The Grothendieck group $K_{0}(\mathcal{B})$ of an abelian category $\mathcal{B}$ is generated by symbols $[M]$, where $M \in O b(\mathcal{B})$, subject to the defining relations $\left[M_{2}\right]=\left[M_{1}\right]+\left[M_{3}\right]$ whenever there is a short exact sequence $0 \longrightarrow M_{1} \longrightarrow M_{2} \longrightarrow M_{3} \longrightarrow 0$. An exact functor $F: \mathcal{A} \rightarrow \mathcal{B}$ between abelian categories induces a homomorphism $[F]: K_{0}(\mathcal{A}) \rightarrow K_{0}(\mathcal{B})$ of Grothendieck groups. Let $A$ be a ring which is free as an abelian group, and $\mathbf{a}=\left\{a_{i}\right\}_{i \in I}$ a basis of $A$, such that $a_{i} a_{j}=\sum_{k} c_{i j}^{k} a_{k}$, where all $c_{i j}^{k}$ are non-negative integers. Furthermore, let $B$ be an $A$-module.

Definition 1. A (weak) abelian categorification of $(A, \mathbf{a}, B)$ consists of an abelian category $\mathcal{B}$, an isomorphism $K_{0}(\mathcal{B}) \cong B$ of abelian groups, and exact endofunctors $F_{i}: \mathcal{B} \longrightarrow \mathcal{B}, i \in I$, such that there are isomorphisms $F_{i} F_{j} \cong \bigoplus_{k} F_{k}^{c_{i j}^{k}}$ of functors for all $i, j \in I$, and for all $i \in I$ the diagram below commutes.

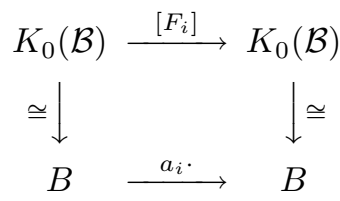

Of course, the existence of a categorification of $B$ implies that there must be a basis of $B$ such that the structure constants are all non-negative integers. Several 
known results in the literature can be considered as categorifications of modules over various rings, see for example $[\mathrm{BFK}],[\mathrm{CR}],[\mathrm{FKS}]$ and references therein.

If $R$ is a finite-dimensional algebra over a field, the Grothendieck group $K_{0}(R-\bmod )$ of the category of finite-dimensional $R$-modules is a finite rank free abelian group with the distinguished basis given by images of simple $R$-modules. The group $K_{0}(R-\bmod )$ has a subgroup $K_{0}^{P}(A-\bmod )$, generated by all $[Q]$ where $Q$ is a projective $R$-module. Note that $K_{0}^{P}(R-\bmod )$ has the distinguished spanning set given by images of indecomposable projective $R$-modules. If the global dimension of $R$ is finite, we have $K_{0}(R-\bmod )=K_{0}^{P}(R-\bmod )$, but in general there is only the obvious inclusion $K_{0}^{P}(R-\bmod ) \subset K_{0}(R-\bmod )$. For the algebras we consider here, this inclusion becomes an isomorphism after tensoring with $\mathbb{Q}$.

Definition 1 also makes sense with $K_{0}^{P}$ in place of $K_{0}$, assuming that the functors $F_{i}$ take projectives to projectives.

\section{Partitions, Specht modules, and category $\mathcal{O}$}

Let $n$ be a positive integer and $S_{n}$ the symmetric group of order $n !$. It is generated by the transpositions $s_{i}=(i, i+1), i=1, \ldots, n-1$. Let $\mathbb{Z}\left[S_{n}\right]$ be the integral group algebra of $S_{n}$. For any partition $\lambda$ of $n$, i.e. $\lambda=\left(\lambda_{1}, \ldots, \lambda_{k}\right), \lambda_{1}+\cdots+\lambda_{k}=n, \lambda_{i} \in$ $\mathbb{N}, \lambda_{1} \geq \lambda_{2} \geq \ldots$, we denote by $\mathcal{S}(\lambda)$ the right Specht $\mathbb{Z}\left[S_{n}\right]$-module corresponding to $\lambda$. Note that these Specht modules give rise to a complete list of irreducible $S_{n}$-modules over the field $\mathbb{C}$. Consider the Lie algebra $\mathfrak{s l}_{n}(\mathbb{C})$ with the standard triangular decomposition $\mathfrak{s l}_{n}(\mathbb{C})=\mathfrak{n}_{-} \oplus \mathfrak{h} \oplus \mathfrak{n}_{+}$. Denote by $\mathcal{O}_{0}$ the corresponding principal block of the BGG-category $\mathcal{O}$ for $\mathfrak{s l}_{n}(\mathbb{C})$, see $[\mathrm{BGG}]$. The simple modules in this category are highest weight modules, with highest weights contained in a certain $S_{n}$-orbit. In particular, the simple objects in $\mathcal{O}_{0}$ are indexed by $w \in S_{n}$ (we assume that the identity element in $S_{n}$ indexes the one-dimensional simple module in $\left.\mathcal{O}_{0}\right)$. For $w \in S_{n}$ we denote by $L(w), \Delta(w)$ and $P(w)$ the corresponding simple, Verma and projective modules in $\mathcal{O}_{0}$, , respectively.

Let $\mu=\left(\mu_{1}, \ldots, \mu_{k}\right)$ be a composition of $n$, obtained by permuting the components of $\lambda$. Associated with it we have the parabolic subalgebra $\mathfrak{p}_{\mu}$ consisting of $\mu$ block upper-triangular matrices in $\mathfrak{s l}_{n}$, and a subgroup $S_{\mu}=S_{\mu_{1}} \times S_{\mu_{2}} \times \cdots \times S_{\mu_{n}}$ of $S_{n}$ as follows. The algebra $\mathfrak{p}_{\mu}$ contains the Borel algebra $\mathfrak{h} \oplus \mathfrak{n}_{+}$; the Levi factor of $\mathfrak{p}_{\mu}$ is the reductive Lie algebra of traceless matrices in $\left(\mathfrak{g l}_{\mu_{1}}(\mathbb{C}) \oplus \cdots \oplus \mathfrak{g l}_{\mu_{k}}(\mathbb{C})\right)+\mathfrak{h} \subset$ $\mathfrak{g l}_{n}$. The group $S_{\mu}$ is the Weyl group of the subalgebra $\mathfrak{s l}_{\mu_{1}}(\mathbb{C}) \oplus \cdots \oplus \mathfrak{s l}_{\mu_{k}}(\mathbb{C})$.

We consider the principal block $\mathcal{O}_{0}^{\mu}$ of the parabolic subcategory of $\mathcal{O}_{0}$ associated with $\mathfrak{p}_{\mu}$ (see $\left.[\mathrm{R}-\mathrm{C}]\right)$. By definition this is the full subcategory given by all objects $M \in \mathcal{O}_{0}$ such that any $m \in M$ is contained in a finite-dimensional $\mathfrak{p}_{\mu}$-stable subspace of $M$ (such modules are called $\mathfrak{p}_{\mu}$-locally finite). The simple objects in $\mathcal{O}_{0}^{\mu}$ are exactly the simple objects in $\mathcal{O}_{0}$ which correspond to the shortest length representatives in the coset $S_{\mu} \backslash S_{n}$. We denote the latter set by $S(\mu)$ and let $w_{\mu}$ denote the longest element in this set. For $w \in S(\mu)$ we denote by $L^{\mu}(w), \Delta^{\mu}(w)$ and $P^{\mu}(w)$ the corresponding simple, generalized Verma and projective modules in $\mathcal{O}_{0}^{\mu}$, respectively. Remark that $L^{\mu}(w)=L(w)$ for $w \in S(\mu)$.

To a finite-dimensional $\mathfrak{s l}_{n}$-module $E$ we associate the functor $F_{E}: \mathcal{O}_{0} \rightarrow \mathcal{O}_{0}$ given by first tensoring with $E$ and then taking the largest direct summand contained in $\mathcal{O}_{0}$. In $[\mathrm{BG}]$, the indecomposable direct summands of such functors were classified (up to isomorphism). They are in natural bijection with the elements of $S_{n}$, where $w \in S_{n}$ corresponds to the indecomposable functor $\theta_{w}$ which maps 
$P(e)$ to $P(w)$. For $i=1, \ldots, n-1$ we abbreviate $\theta_{i}=\theta_{s_{i}}: \mathcal{O}_{0} \rightarrow \mathcal{O}_{0}$. This is the famous translation functor through the $s_{i}$-wall. All the functors $\theta_{w}$ are exact and, by definition, preserve $\mathcal{O}_{0}^{\mu}$. The translations $\theta_{i}$ through walls are self-adjoint functors.

For $w \in S_{n}$ let $\mathcal{R}(w)$ be the right cell of the element $w$ (see for example [KL], or [Do] for a characterization of $\mathcal{R}(w))$. According to the main result of [Ir], the modules $P^{\mu}(w), w \in \mathcal{R}\left(w_{\mu}\right)$, constitute an exhaustive list of indecomposable projectiveinjective modules in $\mathcal{O}_{0}^{\mu}$.

\section{A categorification of the Specht modules}

Let $\mathcal{H}_{n}$ denote the Hecke algebra of $S_{n}$ over $\mathbb{Z}\left[v, v^{-1}\right]$ with the $\mathbb{Z}\left[v, v^{-1}\right]$-basis $H_{w}, w \in W$, and the relations $H_{s}^{2}=H_{e}+\left(v-v^{-1}\right) H_{s}, H_{s} H_{t}=H_{t} H_{s}$ if $s t=t s$, and $H_{s} H_{t} H_{s}=H_{t} H_{s} H_{t}$ if sts $=t s t$, where $s, t \in\left\{s_{i} \mid 1 \leq i<n\right\}$. The algebra $\mathcal{H}_{n}$ is a deformation of $\mathbb{Z}\left[S_{n}\right]$. Furthermore, let $\underline{H}_{w}, w \in W$, denote the KazhdanLusztig basis elements of $\mathcal{H}_{n}$ introduced in [KL], written in the normalization of [So]. The elements of $\underline{H}_{w}, w \in W$, specialize (via $v=1$ ) to the Kazhdan-Lusztig basis elements $\underline{\hat{H}}_{w}$ of $\mathbb{Z}\left[S_{n}\right]$.

Set $P_{\mu}=\bigoplus_{w \in \mathcal{R}\left(w_{\mu}\right)} P^{\mu}(w)$ and denote by $\mathcal{C}_{\mu}$ the full subcategory of $\mathcal{O}_{0}^{\mu}$ which consists of all $M$ admitting a two-step resolution,

$$
P_{1} \rightarrow P_{0} \rightarrow M \rightarrow 0,
$$

with $P_{1}, P_{0} \in \operatorname{add}\left(P_{\mu}\right)$. By $\left[\mathrm{Au}\right.$, Section 5], the category $\mathcal{C}_{\mu}$ is equivalent to $A_{\mu}-\bmod$, where $A_{\mu}=\operatorname{End}_{\mathcal{O}_{0}^{\mu}}\left(P_{\mu}\right)$, in particular, $\mathcal{C}_{\mu}$ is an abelian category. Since projective functors preserve both projectivity and injectivity, they preserve $\operatorname{add}\left(P_{\mu}\right)$. Now from (1) it follows that they preserve $\mathcal{C}_{\mu}$ as well.

Theorem 1. The action of $\theta_{w}, w \in W$, on $\mathcal{C}_{\mu}$ defines on $K_{0}\left(\mathcal{C}_{\mu}\right)$ the structure of an $S_{n}$-module. The restriction of this action to $K_{0}^{P}\left(\mathcal{C}_{\mu}\right)$ gives rise to a categorifcation of the triple $\left(\mathbb{Z}\left[S_{n}\right],\left\{\underline{\hat{H}}_{w}: w \in W\right\}, \mathcal{S}\left(\lambda^{\prime}\right)\right)$, such that the Kazhdan-Lusztig basis in $\mathcal{S}\left(\lambda^{\prime}\right)$ corresponds to the basis given by the isomorphism classes of the indecomposable projective modules, and the action of $\theta_{w}, w \in W$, corresponds to the elements $\underline{\hat{H}}_{w}$ of $\mathbb{Z} S_{n}$.

The associative algebras that correspond to both $\mathcal{O}_{0}$ and $\mathcal{O}_{0}^{\mu}$ are Koszul (see [BGS]) and admit a canonical positive grading (the Koszul grading), which we fix. This allows us to consider graded versions of both $\mathcal{O}_{0}$ and $\mathcal{O}_{0}^{\mu}$; see [St1] for details. If some confusion can arise, to emphasize the graded versions, we will add the superscript $g r$. Let $A_{\mu}-\operatorname{gmod}$ denote the category of all finitely generated graded $A_{\mu}$-modules, where $A_{\mu}$ is equipped with the grading induced from the Koszul grading mentioned above. We denote by $\langle 1\rangle A_{\mu}-\operatorname{gmod} \rightarrow A_{\mu}-\operatorname{gmod}$ the functor that increases the grading by 1 .

In [St1] it was shown that simple modules, Verma modules, generalized Verma modules and projective modules in both $\mathcal{O}_{0}$ and $\mathcal{O}_{0}^{\mu}$ are gradable and, moreover, that the functors $\theta_{i}$ are gradable as well. This allows us to fix, for each $w \in W$, the standard graded lift $\theta_{w}: A_{\mu}-\operatorname{gmod} \rightarrow A_{\mu}-\operatorname{gmod}$ of the functor $\theta_{w}$.

Let $\tilde{\mathcal{S}}(\lambda)$ be the (right) Specht $\mathcal{H}_{n}$-module, which corresponds to $\lambda$ (see e.g. [Mu] or $[\mathrm{KL}])$.

Theorem 2. The graded functors $\theta_{w}, w \in W$, and the functors $\langle 1\rangle$ and $\langle-1\rangle$ induce endofunctors of $\mathcal{C}_{\mu}^{g r}$ and define on $K_{0}\left(\mathcal{C}_{\mu}^{g r}\right)$ the structure of an $S_{n}$-graph. 
The restriction of this action to $K_{0}^{P}\left(\mathcal{C}_{\mu}^{g r}\right)$ gives rise to a categorification of the triple $\left(\mathcal{H}_{n},\left\{\underline{H}_{w}, v, v^{-1}: w \in W\right\}, \tilde{\mathcal{S}}\left(\lambda^{\prime}\right)\right)$, where the Kazhdan-Lusztig basis of $\tilde{\mathcal{S}}\left(\lambda^{\prime}\right)$ corresponds to the basis given by the iso-classes of the indecomposable projective modules with head concentrated in degree zero, and the action of $\theta_{w}$ (resp. $\langle 1\rangle$ ) corresponds to the action of $\underline{H}_{w}$ (resp. of $\left.v\right)$.

Proof of Theorem 1 and Theorem 2. Theorem 1 is obtained from Theorem 2 via evaluation $v=1$, hence it is enough to prove Theorem 2. As a consequence of the Kazhdan-Lusztig theorem and [St1, Theorem 5.1], for $w \in S_{n}$ we have the following equality in $K_{0}\left(\mathcal{O}_{0}^{g r}\right)$ :

$$
\left[\theta_{i} L(w)\right]= \begin{cases}0 & w s_{i}>w \\ {[L(w)\langle 1\rangle]+[L(w)\langle-1\rangle]+\sum_{w^{\prime} \in S_{n}} \mu\left(w^{\prime}, w\right)\left[L\left(w^{\prime}\right)\right]} & w s_{i}<w\end{cases}
$$

where $\mu\left(w^{\prime}, w\right)$ denotes the Kazhdan-Lusztig $\mu$-function; see [KL, Definition 1.2]. If $w \in \mathcal{R}\left(w_{\mu}\right)$, then we get the following equality for the induced action on $K_{0}\left(\mathcal{C}_{\mu}^{g r}\right)$ :

$$
\left[\theta_{i} L^{\mu}(w)\right]= \begin{cases}0 & w s_{i}>w \\ {\left[L^{\mu}(w)\langle 1\rangle\right]+\left[L^{\mu}(w)\langle-1\rangle\right]+\sum_{w^{\prime} \in \mathcal{R}\left(w_{\mu}\right)} \mu\left(w^{\prime}, w\right)\left[L^{\mu}\left(w^{\prime}\right)\right]} & w s_{i}<w\end{cases}
$$

This means that the action of $\theta_{i}$ and $\langle-1\rangle$ on $\mathcal{C}_{\mu}^{g r}$ defines on $K_{0}\left(\mathcal{C}_{\mu}^{g r}\right)$ the structure of an $S_{n}$-graph. By [KL, Theorem 1.4], we obtain a simple $\mathcal{H}_{n}$-module over any field of characteristic 0 . So, to complete the proof we just have to show that this module is exactly $\tilde{\mathcal{S}}\left(\lambda^{\prime}\right)$. And to do this we can specialize to $v=1$. In this case the necessary statement follows from [Na, Theorem 4.1].

Finally, let $c_{w, w^{\prime}}^{w^{\prime \prime}}$ be the structure constants of $\mathcal{H}_{n}$ with respect to the KazhdanLusztig basis, that is

$$
\underline{H}_{w} \underline{H}_{w^{\prime}}=\sum_{w^{\prime \prime} \in S_{n}} c_{w, w^{\prime}}^{w^{\prime \prime}} \underline{H}_{w^{\prime \prime}}
$$

Then $c_{w, w^{\prime}} \in \mathbb{Z}\left[v, v^{-1}\right]$ and the existence of an isomorphism

$$
\theta_{w} \theta_{w^{\prime}} \cong \sum_{w^{\prime \prime} \in S_{n}} c_{w, w^{\prime}}^{w^{\prime \prime}} \theta_{w^{\prime \prime}}
$$

follows from [BG, Theorem 3.4(iv)], the Kazhdan-Lusztig theorem and [St1, Theorem 5.1].

Example 1. The trivial representation. If $\mu=(1,1,1, \ldots, 1)$, then $\mathcal{O}_{0}^{\mu}=\mathcal{O}_{0}$. There is a unique indecomposable projective-injective module $P\left(w_{0}\right)$, where $w_{0}$ is the longest element in $S_{n}$. We have $\theta_{i} P\left(w_{0}\right) \cong P\left(w_{0}\right)\langle 1\rangle \oplus P\left(w_{0}\right)\langle-1\rangle$, which implies that $\left[\theta_{i}\right]\left[L\left(w_{0}\right)\right]=\left[L\left(w_{0}\right)\langle 1\rangle\right]+\left[L\left(w_{0}\right)\langle-1\rangle\right]$. Hence $K_{0}\left(\mathcal{C}_{\mu}^{g r}\right) \cong \mathbb{Z}\left[v, v^{-1}\right]$ and also $K_{0}^{P}\left(\mathcal{C}_{\mu}^{g r}\right) \cong \mathbb{Z}\left[v, v^{-1}\right]$ (note that $K_{0}^{P}\left(\mathcal{C}_{\mu}^{g r}\right) \subsetneq K_{0}\left(\mathcal{C}_{\mu}^{g r}\right)$ in this case); the action of $H_{i}+v$ is just the multiplication by $v+v^{-1}$. This construction therefore categorifies the trivial representation of the symmetric group and its Hecke algebra deformation.

We remark that in this case the endomorphism algebra $A_{\mu}$ of $P\left(w_{0}\right)$ is isomorphic to the complex cohomology ring of the full flag variety of $\mathbb{C}^{n}$, and $\mathcal{C}_{\mu}^{g r}$ is equivalent to the category of graded finite-dimensional modules over this algebra. Further, $\theta_{i}$ is isomorphic to the functor of tensoring with the graded $A_{\mu}$-bimodule which is the cohomology ring of an iterated flag variety (having two subspaces of dimension $i$ ). 
Example 2. The sign representation. If $\mu=(n)$, then $\mathcal{O}_{0}^{\mu}$ is semi-simple and contains a unique, up to isomorphism, simple projective (and hence also injective) module, namely the trivial module $L(e)$. We have $\theta_{i} L(e)=0$, so that $K_{0}\left(\mathcal{C}_{\mu}\right)=$ $K_{0}^{P}\left(\mathcal{C}_{\mu}\right) \cong \mathbb{Z}\left[v, v^{-1}\right]$ and $H_{i}+v$ acts by 0 . We obtain a categorification of the sign representation of the symmetric group and its Hecke algebra deformation.

Remark 3. The action of projective functors on (the graded version of) $\mathcal{O}_{0}$ categorifies the regular representation of $S_{n}$ (respectively $\mathcal{H}_{n}$ ). This is a straightforward calculation using the basis $[\Delta(w)], w \in S_{n}$, of $K_{0}\left(\mathcal{O}_{0}\right)$. The basis $[P(w)], w \in S_{n}$, then corresponds to the Kazhdan-Lusztig basis in $\mathbb{Z}\left[S_{n}\right]$ (respectively $\mathcal{H}_{n}$ ).

\section{THE BILINEAR FORMS}

Now recall that every Specht module $\tilde{\mathcal{S}}(\lambda)$ has a symmetric, non-degenerate, $\mathcal{H}_{n^{-}}$ invariant bilinear form $\langle$,$\rangle with values in \mathbb{Z}\left[v, v^{-1}\right]$ which is unique up to a scalar $[\mathrm{Mu}$, page 114]. We give a categorical interpretation of this form. For any $\mathbb{Z}$-graded complex vector space $M=\bigoplus_{j \in \mathbb{Z}} M^{j}$ let $h(M)=\sum_{j \in \mathbb{Z}}\left(\operatorname{dim}_{\mathbb{C}} M^{j}\right) v^{j} \in \mathbb{Z}\left[v, v^{-1}\right]$ be the corresponding Hilbert polynomial.

Consider $A_{\mu}-$ gmod, the graded version of $\mathcal{C}_{\mu}$. If $M, N \in A_{\mu}-$ gmod, then the space $E^{i}(M, N):=\operatorname{Ext}_{A_{\mu}-\bmod }^{i}(M, N)$ is a $\mathbb{Z}$-graded vector space for any $i \in \mathbb{Z}$. Set $h(E(M, N))=\sum_{i \in \mathbb{Z}}(-1)^{i} h\left(E^{i}(M, N)\right)$. Let d denote the graded lift of the standard duality on $\mathcal{O}_{0}^{\mu}$, restricted to the category $\mathcal{C}_{\mu}^{g r}$ (see [St1]).

The following result categorifies the bilinear forms on Specht modules.

Proposition 1. Let $\mu$ be a composition of $n$ and $\lambda$ be the corresponding partition. Then

$$
\beta\left({ }_{-},-\right):=h\left(E\left(_{-}, \mathrm{d}\left({ }_{-}\right)\right)\right): \operatorname{Ob}\left(A_{\mu}-\operatorname{gmod}\right) \times \mathrm{Ob}\left(A_{\mu}-\operatorname{gmod}\right) \rightarrow \mathbb{Q}(v)
$$

descends to a symmetric, non-degenerate, $\mathcal{H}_{n}$-invariant bilinear form $\langle\cdot, \cdot\rangle$ on $K_{0}\left(A_{\mu}-\right.$ gmod $)$ and $K_{0}^{P}\left(A_{\mu}\right.$-gmod $)$ with values in $\mathbb{Q}(v)$ and $\mathbb{Z}\left[v, v^{-1}\right]$, respectively.

Proof. The form is bilinear, since the bifunctor $\beta$ is additive in both arguments. To verify the symmetry, it is therefore enough to check that $\beta(M, N)=\beta(N, M)$, when $M, N$ are projective (and therefore injective). We have $\beta(M, N)=\beta(\mathrm{d}(N), \mathrm{d}(M))$ $=\beta(N, M)$, since $\mathrm{d}(M) \cong M\langle k\rangle$ and $\mathrm{d}(N) \cong N\langle k\rangle$ for some $k \in \mathbb{Z}$, which is the same for both $M$ and $N$ by [MS, Theorem 5.2(1)]. So, the form is symmetric. To see that the form is $\mathcal{H}_{n}$-invariant we only have to verify that $\beta\left(P^{\mu}(x), \theta_{i} P^{\mu}(w)\right)=$ $\left(v+v^{-1}\right) \beta\left(P^{\mu}(x), P^{\mu}(w)\right)$ for any $x, w \in S_{n}$ such that $x s_{i}<x$ and $w s_{i}<w$ in the Bruhat ordering ([Fu, Proposition 6.1]). However, this is clear, since $\theta_{i} P^{\mu}(w) \cong$ $P^{\mu}(w)\langle 1\rangle \oplus P^{\mu}(w)\langle-1\rangle$ (this follows from [St1, Theorem 5.1]).

To see that it is non-degenerate (i.e. that it has trivial radical), we observe that in the basis given by indecomposable projectives with heads concentrated in degree zero, the form is given by the matrix $M=h\left(\operatorname{Hom}_{A_{\mu}}\left({ }_{-}, \mathrm{d}\left({ }_{-}\right)\right)\right)$. Since the algebra $A_{\mu}$ is positively graded, it follows that the entries off the diagonal of $M$ are in $v \mathbb{Z}[v]$, and the ones on the diagonal are of the form $1+f$, where $f \in v \mathbb{Z}[v]$. Hence, the matrix $M$ has a non-zero determinant and thus the form is non-degenerate.

It is left to note that the algebra $A_{\mu}$ has infinite global dimension in general, and hence $h(E(M, N))$ is a power series and not a Laurent polynomial for general $M$ and $N$. However, since the (graded) Cartan matrix of $A_{\mu}$ is invertible, it follows that for simple $M$ and $N$ we can compute $h(E(M, N))$ by inverting the Cartan matrix and hence $h(E(M, N))$ can be written as a rational function. 


\section{REFERENCES}

[Au] M. Auslander, Representation theory of Artin algebras. I, II. Comm. Algebra 1 (1974), 177-268; ibid. 1 (1974), 269-310. MR0349747 (50:2240)

[BGS] A. Beilinson, V. Ginzburg and W. Soergel, Koszul duality patterns in representation theory, J. Amer. Math. Soc. 9 (1996), no. 2, 473-527. MR1322847 (96k:17010)

[BG] J. Bernstein and S. Gelfand, Tensor products of finite and infinite dimensional representations of semi-simple Lie algebras, Comp. Math. 41 (1980), 245-285. MR581584 (82c:17003)

[BFK] J. Bernstein, I. B. Frenkel and M. Khovanov, A categorification of the Temperley-Lieb algebra and Schur quotients of $U\left(\mathfrak{s l}_{2}\right)$ via projective and Zuckerman functors, Selecta Math. (N.S.) 5 (1999), no.2, 199-241. MR1714141 (2000i:17009)

[BGG] I. N. Bernstein, I. M. Gelfand and S. I. Gelfand, A certain category of $\mathfrak{g}$-modules, Funkcional. Anal. i Priložen. 10 (1976), no. 2, 1-8. MR0407097 (53:10880)

[CR] J. Chuang and R. Rouquier, Derived equivalences for symmetric groups and $s l_{2}-$ categorification, to appear in Annals Math., math.RT/0407205.

[Do] J. M. Douglass, An involution of the variety of flags fixed by a unipotent linear transformation, Adv. in Appl. Math. 17 (1996), no. 3, 357-379. MR1406407 (98c:14042)

[FKS] I. B. Frenkel, M. Khovanov and C. Stroppel, A categorification of finite-dimensional irreducible representations of quantum $s l(2)$ and their tensor products, Selecta Math. 12 (2006) nos. 3-4, 379-431. MR2305608 (2008a:17014)

[Fu] F. Fung, On the topology of components of some Springer fibers and their relation to Kazhdan-Lusztig theory. Adv. Math. 178 (2003), no. 2, 244-276. MR1994220 (2004m:20087)

[Ir] R. S. Irving, Projective modules in the category $\mathcal{O}_{S}$ : self-duality, Trans. Amer. Math. Soc. 291 (1985), no. 2, 701-732. MR800259 (87i:17005)

[Ja] J. C. Jantzen, Moduln mit einem höchsten Gewicht, Lecture Notes in Mathematics, 750, Berlin, (1979). MR552943 (81m:17011)

[KL] D. Kazhdan and G. Lusztig, Representations of Coxeter groups and Hecke algebras, Invent. Math. 53 (1979), no. 2, 165-184. MR560412 (81j:20066)

[MS] V. Mazorchuk and C. Stroppel, Projective-injective modules, Serre functors and symmetric algebras, to appear in J. reine angew. Math. math.RT/0508119.

[Mu] G. E. Murphy, The representations of Hecke algebras of type $A_{n}$, J. Algebra 173 (1995), no. 1, 97-121. MR1327362 (96b:20013)

[Na] H. Naruse, On an isomorphism between Specht module and left cell of $\mathfrak{S}_{n}$, Tokyo J. Math. 12 (1989), no. 2, 247-267. MR1030495 (90k:20025)

[R-C] A. Rocha-Caridi, Splitting criteria for $\mathfrak{g}$-modules induced from a parabolic and the BGG resolution of a finite-dimensional, irreducible $\mathfrak{g}$-module. Trans. Amer. Math. Soc. 262 (1980), no. 2, 335-366. MR586721 (82f:17006)

[So] W. Soergel, Kazhdan-Lusztig polynomials and a combinatorics for tilting modules. Represent. Theory 1 (1997), 83-114. MR1444322 (98d:17026)

[St1] C. Stroppel, Category $\mathcal{O}$ : gradings and translation functors, J. Algebra 268 (2003), no. 1, 301-326. MR2005290 (2004i:17007)

[St2] C. Stroppel, Perverse sheaves on Grassmannians, Springer fibres and Khovanov homology, math.RT/0608234.

Department of Mathematics, Columbia University, New York, New York

E-mail address: khovanov@math.columbia.edu

Department of Mathematics, Uppsala University, Uppsala, Sweden

E-mail address: mazor@math.uu.se

Department of Mathematics, University of Glasgow, Glasgow, United Kingdom

E-mail address: c.stroppel@maths.gla.ac.uk 